Fern, Florian; FüßI, Roland; Eichfelder, Gabriele; Manske, Eberhard;

Kühnel, Michael:

Coordinate transformation and its uncertainty under consideration of a non orthogonal coordinate base

Original published in: $\quad$ Measurement science and technology. - Bristol : IOP Publ.. - 32 (2021), 4, art. 045001, 6 pp.

Original published: $\quad$ 2021-01-11

ISSN: $\quad$ 1361-6501

DOI: $\quad$ 10.1088/1361-6501/aba3f5

[Visited: 2021-02-22]

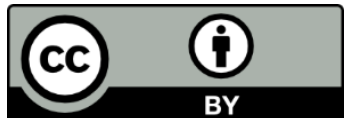

This work is licensed under a Creative Commons Attribution 4.0 International license. To view a copy of this license, visit https://creativecommons.org/licenses/by/4.0/ 


\title{
Coordinate transformation and its uncertainty under consideration of a non-orthogonal coordinate base
}

\author{
Florian Fern ${ }^{\circledR}$, Roland FüßI, Gabriele Eichfelder, Eberhard Manske \\ and Michael Kühnel \\ Institute of Process Measurement and Sensor Technology, Technische Universität Ilmenau, Germany \\ E-mail: florian.fern@tu-ilmenau.de
}

Received 24 March 2020, revised 29 June 2020

Accepted for publication 8 July 2020

Published 11 January 2021

\begin{abstract}
Nanopositioning and nanomeasuring machines are 3D coordinate measuring systems with nanometer precision at measurement volumes in the cubic centimeter range whose coordinate base is formed by an interferometer system with a common mirror corner. This mirror corner shows a typical deviation from the orthogonal coordinate base of up to $17 \mu \mathrm{rad}$ with an uncertainty of $0.07 \mu \mathrm{rad}$. After a brief description of nanomeasuring and nanopositioning machines, a transformation model from the skewed coordinate system into the orthogonal coordinate system is determined. Starting from this complete transformation model, the result of model simplifications on the transformation behaviour is analysed and discussed. A GUM-based uncertainty calculation shows that the linearised coordinate transformation does not increase the error and the uncertainty significantly.
\end{abstract}

Keywords: metrology, nano-CMM, uncertainty, coordinate transformation, NPMM-200

\section{Nanomeasuring and nanopositioning machines}

In recent years, different concepts and designs of nano coordinate measuring machines have been discussed and successfully implemented [1-4]. The nanopositioning and nanomeasuring machine NPMM-200 [5] was developed at the TU-Ilmenau. It works in the sample scanning mode, where the sample is moved on a stage relative to the fixed sensor on an $x$-, $y$-, and $z$-stage with a working volume of $200 \mathrm{~mm} \times 200 \mathrm{~mm} \times$ $25 \mathrm{~mm}$. With frequency-stabilized He-Ne laser-based Michelson interferometers the position and the angular deviations of the stage are measured and closed-loop controlled with a length resolution of $0.016 \mathrm{~nm}$. The interferometer beams used for the length measurement cross virtually and perpendicular to in the measurement point of the sensor, which is called the

Original Content from this work may be used under the terms of the Creative Commons Attribution 4.0 licence. Any further distribution of this work must maintain attribution to the author(s) and the title of the work, journal citation and DOI.
Abbe-point [6]. On the moving stage itself a 3D mirror corner is fixed. This mirror corner is a monolithic element made of Zerodur with three reflective flats in the $x$-, $y$ - and $z$-direction (see figures 1 and 2) which are the measuring reflector for the used interferometers.

\subsection{Uncertainty model}

In order to achieve a measurement uncertainty in the low nanometre level the complete machine as well as the environmental disturbances must be described precisely. A vectorbased uncertainty model has turned out to be a very successful way to find the model equations for nano measuring machines $[7,8]$. Thereby, the difference in position (the distance) between two measured points is described by two closed vector chains. Those vector chains consist of different subvectors. Every subvector describes one effect by an equation, for example the influence of the change in the refractive index of air, thermal expansion, or the deviations of the orthogonality of the mirror corner. Thereby, the non-orthogonality of the mirror corner has been described up to now with a simplified coordinate transformation. 


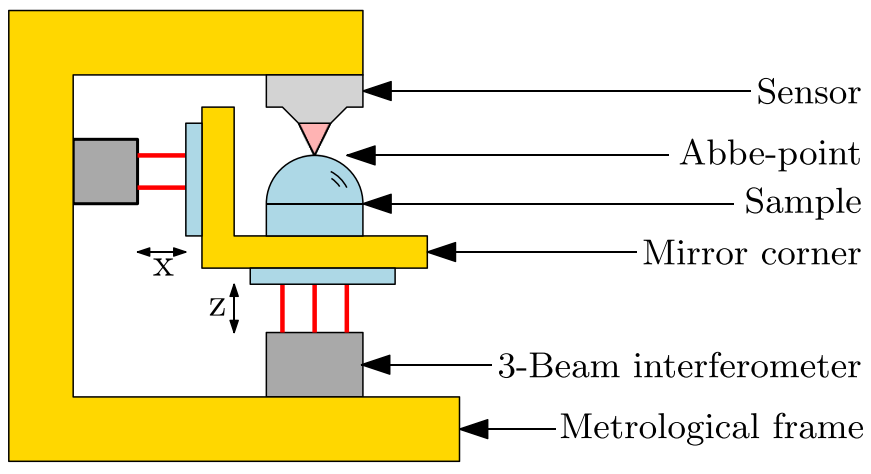

Figure 1. 2D model of the NPMM-200 working principle in the $x$ and $z$-direction. The mirror corner, carrying the sample, moves relative to the sensor in a working volume of $200 \mathrm{~mm} \times 200 \mathrm{~mm} \times$ $25 \mathrm{~mm}$. The position of the mirror corner, which is fixed to the stage, is measured with three laser interferometers. Angular deviations are measured and corrected with multibeam interferometers.

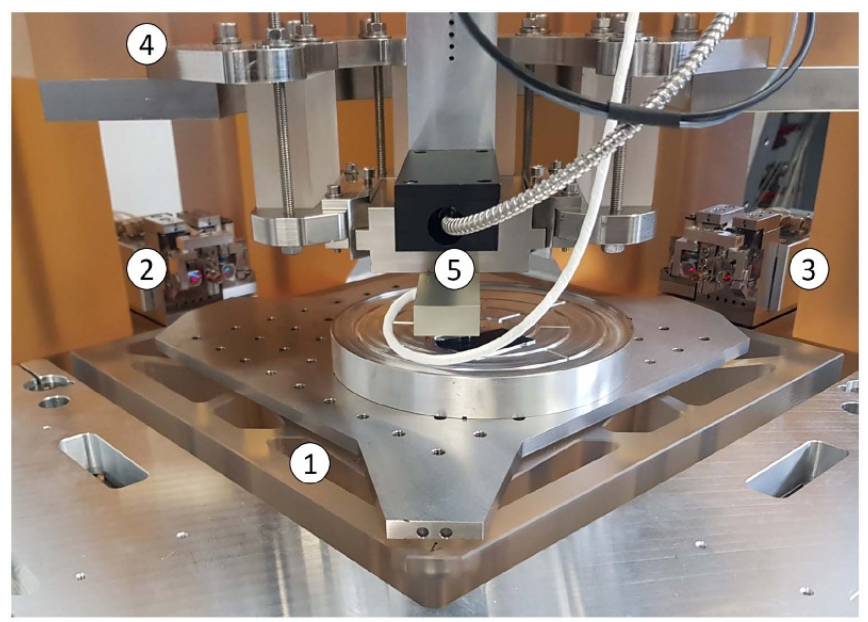

Figure 2. Photograph of the NPMM-200 without the vacuum chamber. (1) Mirror corner (z-mirror is not shown); (2) $y$-interferometer; (3) $x$-interferometer; (4) upper part of the metrological frame; (5) optical surface sensor.

This paper addresses a systematic way to determine the complete coordinate transformation. In the end, different simplifications are compared and the influence on the measurement uncertainty is discussed in detail.

\section{Coordinate system}

The coordinate system of the NPMM-200 is represented by the $x$-, $y$ - and $z$-mirror of the mirror corner and the measurement beams of the interferometers. Due to unavoidable inaccuracies in the manufacture of the mirror corner, these mirrors are not perfectly perpendicular to each other.

These mirrors set up a skewed coordinate system. This skewed coordinate system is described by a set of normed basis vectors $\left\langle\vec{g}_{1}, \vec{g}_{2}, \vec{g}_{3}\right\rangle$ which corresponds to the normal vectors of the mirrors $\left\langle\vec{g}_{\mathrm{x}}, \vec{g}_{\mathrm{y}},-\vec{g}_{\mathrm{z}}\right\rangle$ (see figure 3 ).

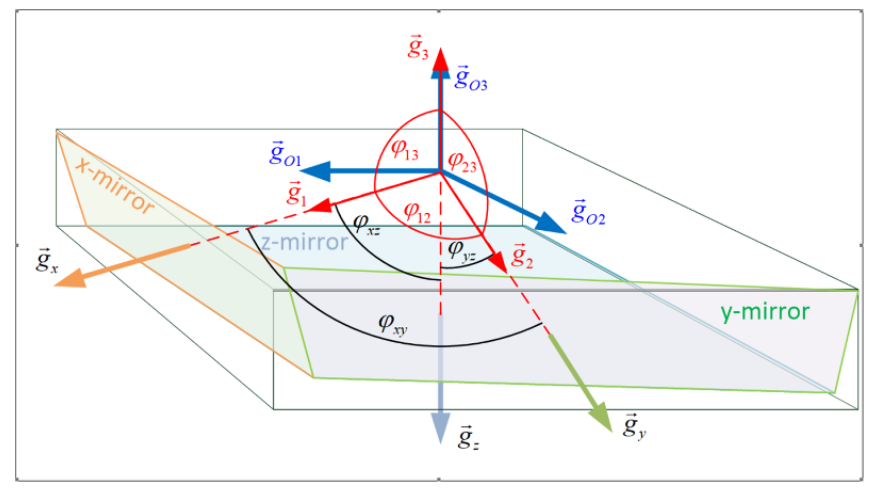

Figure 3. Vector components and vector bases of the non perpendicular mirrors of a mirror corner. $\left\langle\vec{g}_{\mathrm{O} 1}, \vec{g}_{\mathrm{O} 2}, \vec{g}_{\mathrm{O} 3}\right\rangle$ orthonormal vector base; $\left\langle\vec{g}_{1}, \vec{g}_{2}, \vec{g}_{3}\right\rangle$ skewed vector base; $\left\langle\vec{g}_{\mathrm{x}}, \vec{g}_{\mathrm{y}}, \vec{g}_{\mathrm{z}}\right\rangle$ normal vector of the mirrors.

To calculate the coordinates of a measurement point $P$ in the orthonormal coordinate system $\left\langle\vec{g}_{\mathrm{O} 1}, \vec{g}_{\mathrm{O} 2}, \vec{g}_{\mathrm{O} 3}\right\rangle$ the coordinates measured by the interferometers $\left(x_{s} y_{s} z_{s}\right)$ must be converted to coordinates $\left(x_{O} y_{O} z_{O}\right)$ in the orthonormal coordinate system. The vector base of the skewed coordinate system is described as a linear combination of the basis vectors of the orthogonal coordinate system:

$$
\begin{aligned}
& \vec{g}_{1}=a_{1}{ }^{1} \vec{g}_{\mathrm{O} 1}+a_{1}{ }^{2} \vec{g}_{\mathrm{O} 2}+a_{1}{ }^{3} \vec{g}_{\mathrm{O} 3} \\
& \vec{g}_{2}=a_{2}{ }^{1} \vec{g}_{\mathrm{O} 1}+a_{2}{ }^{2} \vec{g}_{\mathrm{O} 2}+a_{2}{ }^{3} \vec{g}_{\mathrm{O} 3} \\
& \vec{g}_{3}=a_{3}{ }^{1} \vec{g}_{\mathrm{O} 1}+a_{3}^{2} \vec{g}_{\mathrm{O} 2}+a_{3}{ }^{3} \vec{g}_{\mathrm{O} 3} .
\end{aligned}
$$

By the Einstein notation [10], this system of equations can be shortened:

$$
\vec{g}_{l}=a_{l}^{m} \vec{g}_{O m} .
$$

The transformation coefficients $a_{l}^{m}$ are the elements of the transformation matrix $\left(a_{l}^{m}\right)$ :

$$
\left(a_{l}^{m}\right)=\left(\begin{array}{lll}
a_{1}^{1} & a_{1}^{2} & a_{1}^{3} \\
a_{2}{ }^{1} & a_{2}^{2} & a_{2}^{3} \\
a_{3}^{1} & a_{3}^{2} & a_{3}^{3}
\end{array}\right) .
$$

Those elements of the transformation matrix can be calculated by means of the angles $\varphi_{12}, \varphi_{13}$ and $\varphi_{23}$ between the basis vectors $\left\langle\vec{g}_{1}, \vec{g}_{2}, \vec{g}_{3}\right\rangle$. The angles $\varphi_{12}, \varphi_{13}$ and $\varphi_{23}$ are derived from the angles between mirror normal vectors $\left\langle\vec{g}_{\mathrm{x}}, \vec{g}_{\mathrm{y}}, \vec{g}_{\mathrm{z}}\right\rangle$ :

$$
\begin{array}{cc}
\varphi_{12} & =\varphi_{x y} \\
\varphi_{13} & =\pi-\varphi_{x z} \\
\varphi_{23} & =\pi-\varphi_{y z} .
\end{array}
$$

The angles $\varphi_{x y}, \varphi_{y z}, \varphi_{x z}$ between the normal vectors of the mirror surfaces can be measured by using a self-compensating 
combination of penta prisms and an electronic autocollimator [9]. All matrix elements can be calculated directly by using the following assumptions:

$$
\begin{gathered}
\left|\vec{g}_{1}\right|=\left|\vec{g}_{2}\right|=\left|\vec{g}_{3}\right|=1 \\
\varphi_{12}=\vec{g}_{1} \cdot \vec{g}_{2} \\
\varphi_{13}=\vec{g}_{1} \cdot \vec{g}_{3} \\
\varphi_{23}=\vec{g}_{2} \cdot \vec{g}_{3} .
\end{gathered}
$$

Therewith the transformation matrix is completely given (see equation (12)).

\section{Interferometrical measured components}

Based on the working principle of the NPMM-200, the interferometers are aligned perpendicular with the mirror surface in order to achieve the maximal reflected laser light.

Hence, the movement of the mirror corner is measured in the direction of the normal vectors of the mirrors. Those measured non-orthogonal covariant coordinates $r_{1}, r_{2}, r_{3}$ must be converted into orthogonal coordinates $r_{O}^{1}, r_{O}^{2}, r_{O}^{3}$ (see figures 4 and 5):

$$
r_{O m}=r_{O}^{m} \quad \text { with } \quad m=1,2,3
$$

It has to be taken into account that in the orthogonal basis vector system, covariant and contravariant components, and therewith the corresponding coordinates, are equal:

$$
\left(a_{l}^{m}\right)=\left(\begin{array}{ccc}
\sqrt{\sin ^{2}\left(\varphi_{13}\right)-\left(\frac{\cos \left(\varphi_{12}\right)-\cos \left(\varphi_{13}\right) \cos \left(\varphi_{23}\right)}{\sin \left(\varphi_{23}\right)}\right)^{2}} & \frac{\cos \left(\varphi_{12}\right)-\cos \left(\varphi_{13}\right) \cos \left(\varphi_{23}\right)}{\sin \left(\varphi_{23}\right)} & \cos \left(\varphi_{13}\right) \\
0 & \sin \left(\varphi_{23}\right) & \cos \left(\varphi_{23}\right) \\
0 & 0 & 1
\end{array}\right)
$$

$$
r_{O m}=r_{O}^{m} \quad \text { with } \quad m=1,2,3 \ldots
$$

\section{Transformation of the non-orthogonal coordinates in orthogonal coordinates}

The covariant non-orthogonal basis vector system $\left\langle\vec{g}_{1}, \vec{g}_{2}, \vec{g}_{3}\right\rangle$ has to be calculated using the orthonormal basis vector system $\left\langle\vec{g}_{\mathrm{O} 1}, \vec{g}_{\mathrm{O} 2}, \vec{g}_{\mathrm{O} 3}\right\rangle$ and the transformation matrix $\left(a_{l}^{m}\right)$ (see equation (4)). The covariant coordinates transform like covariant basis vectors [10]. Hence, it follows according to equation (4):

$$
r_{l}=a_{l}^{m} r_{O m}
$$

Or in matrix notation:

$$
\left(\begin{array}{l}
r_{1} \\
r_{2} \\
r_{3}
\end{array}\right)=\left(\begin{array}{lll}
a_{1}^{1} & a_{1}^{2} & a_{1}^{3} \\
a_{2}^{1} & a_{2}^{2} & a_{2}^{3} \\
a_{3}^{1} & a_{3}^{2} & a_{3}^{3}
\end{array}\right) \cdot\left(\begin{array}{l}
r_{O 1} \\
r_{O 2} \\
r_{O 3}
\end{array}\right) .
$$

The orthogonal coordinates are calculated by using the inverted matrix $A_{\text {cory }}$ :

$$
r_{O m}=\left(a_{l}^{m}\right)^{-1} \cdot\left(r_{l}\right)=A_{\mathrm{cor}} \cdot\left(r_{l}\right) .
$$

This inverted matrix is given in equation (17):

$$
\left(a_{l}^{m}\right)^{-1}=A_{\mathrm{cor}}=\left(\begin{array}{ccc}
\frac{\sin \left(\varphi_{23}\right)}{\sqrt{C}} & \frac{\cos \left(\varphi_{13}\right) \cos \left(\varphi_{23}\right)-\cos \left(\varphi_{12}\right)}{\sqrt{C} \sin \left(\varphi_{23}\right)} & \frac{\cos \left(\varphi_{12}\right) \cos \left(\varphi_{23}\right)-\cos \left(\varphi_{13}\right)}{\sqrt{C} \sin \left(\varphi_{23}\right)} \\
0 & \frac{1}{\sin \left(\varphi_{23}\right)} & -\frac{\cos \left(\varphi_{23}\right)}{\sin \left(\varphi_{23}\right)} \\
0 & 0 & 1
\end{array}\right)
$$

with

$$
\begin{aligned}
C= & 1+2 \cos \left(\varphi_{12}\right) \cos \left(\varphi_{13}\right) \cos \left(\varphi_{23}\right)-\cos ^{2}\left(\varphi_{12}\right) \\
& -\cos ^{2}\left(\varphi_{13}\right)-\cos ^{2}\left(\varphi_{23}\right) .
\end{aligned}
$$

Relating to the interferometer setup the measured nonorthogonal coordinates $r_{1}, r_{2}, r_{3}$ are now referred to $x_{s}, y_{s}, z_{s}$, and the orthogonal coordinates $r_{\mathrm{O} 1}, r_{\mathrm{O} 2}, r_{\mathrm{O} 3}$ are referred to $x_{O}, y_{O}, z_{O}$. With equations (16) and (17) the desired orthogonal 


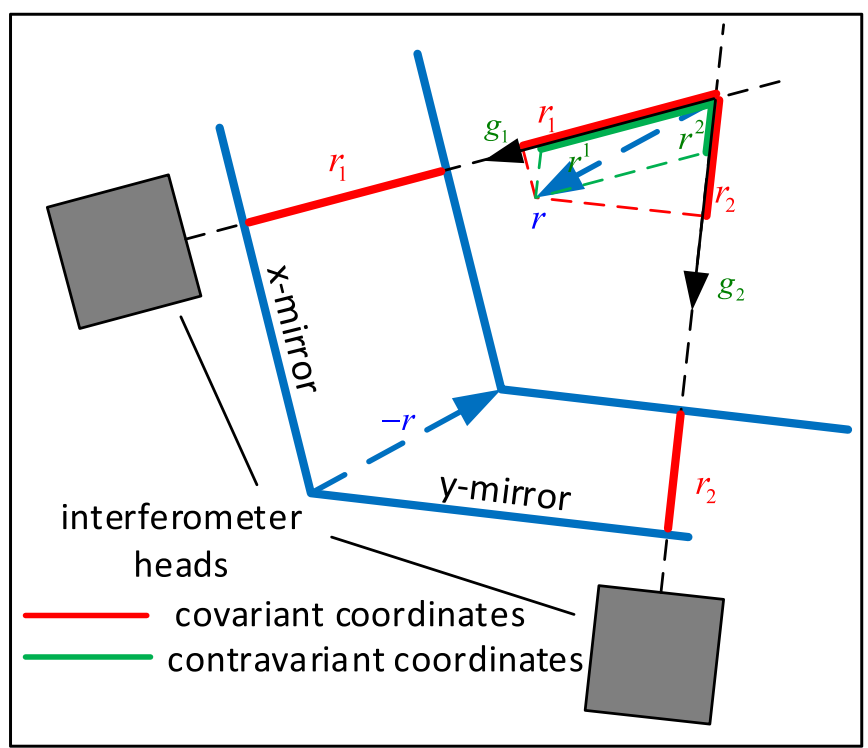

Figure 4. $2 \mathrm{D}$ vector components which are measured by the interferometers used in a nanomeasuring and nanopositioning machine. $\vec{r}$ position vector; $r_{1}, r_{2}$ vector components measured in covariant coordinates .

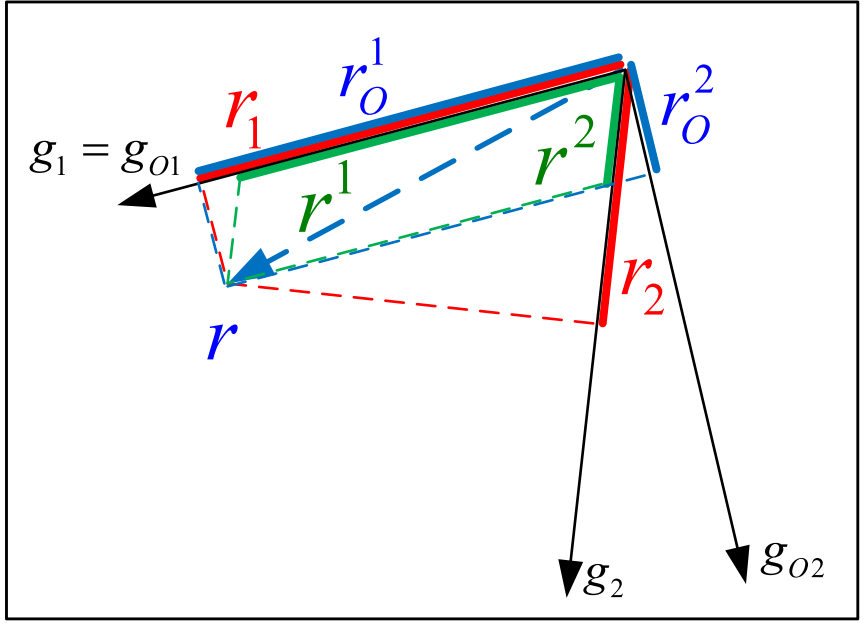

Figure 5. Detailed view on 2D vector components which are measured by the interferometers used in a nanomeasuring and nanopositioning machine (see figure 4).

coordinates can be calculated directly from the measured nonorthogonal coordinates:

$$
\begin{gathered}
x_{O}=\left(x_{s} y_{s} z_{s}\right) \cdot\left(\begin{array}{c}
\frac{\sin \left(\varphi_{23}\right)}{\sqrt{C}} \\
\frac{\cos \left(\varphi_{13}\right) \cos \left(\varphi_{23}\right)-\cos \left(\varphi_{12}\right)}{\sqrt{C} \sin \left(\varphi_{23}\right)} \\
\frac{\cos \left(\varphi_{12}\right) \cos \left(\varphi_{23}\right)-\cos \left(\varphi_{13}\right)}{\sqrt{C} \sin \left(\varphi_{23}\right)}
\end{array}\right), \\
y_{O}=\left(x_{s} y_{s} z_{s}\right) \cdot\left(\begin{array}{c}
0 \\
\frac{1}{\sin \left(\varphi_{23}\right)} \\
-\frac{\cos \left(\varphi_{23}\right)}{\sin \left(\varphi_{23}\right)}
\end{array}\right),
\end{gathered}
$$

$$
z_{O}=\left(x_{s} y_{s} z_{s}\right) \cdot\left(\begin{array}{l}
0 \\
0 \\
1
\end{array}\right)
$$

\section{Simplification and evaluation}

By applying the Taylor approximation to the elements of the transformation matrix $A_{\text {corr }}$ (see equation (17)) and neglecting the higher-order terms, the transformation matrix can be simplified. The angles $\varphi_{i j}$ have a small deviation $\Delta \varphi_{i j}$ from $90^{\circ}$. Hence the trigonometric functions are simplified to

$$
\begin{gathered}
\sin \left(\varphi_{i j}\right) \approx 1 \\
\cos \left(\varphi_{13}\right) \cos \left(\varphi_{23}\right)-\cos \left(\varphi_{12}\right) \approx-\cos \left(\varphi_{12}\right) \\
\cos \left(\varphi_{12}\right) \cos \left(\varphi_{23}\right)-\cos \left(\varphi_{13}\right) \approx-\cos \left(\varphi_{13}\right) \\
\sqrt{C} \approx 1 .
\end{gathered}
$$

By replacing the exact matrix elements with the approximations (see equation (22)) the simplified transformation $A_{\text {sim }}$ is given:

$$
A_{\text {sim }}=\left(\begin{array}{ccc}
1 & -\cos \left(\varphi_{12}\right) & -\cos \left(\varphi_{13}\right) \\
0 & 1 & -\cos \left(\varphi_{23}\right) \\
0 & 0 & 1
\end{array}\right)
$$

With $\quad \varphi_{i j}=0.5 \pi+\Delta \varphi_{i j} \quad$ and $\quad \cos \left(0.5 \pi+\Delta \varphi_{i j}\right)=$ $-\sin \left(\Delta \varphi_{i j}\right) \approx-\Delta \varphi_{i j}$ the transformation matrix can be linearised:

$$
A_{\operatorname{lin}}=\left(\begin{array}{ccc}
1 & \Delta \varphi_{12} & \Delta \varphi_{13} \\
0 & 1 & \Delta \varphi_{23} \\
0 & 0 & 1
\end{array}\right)
$$

The angles given in equation (6),

$$
\begin{aligned}
& \Delta \varphi_{12}=\Delta \varphi_{x y} \\
& \Delta \varphi_{13}=-\Delta \varphi_{x z} \\
& \Delta \varphi_{23}=-\Delta \varphi_{y z}
\end{aligned}
$$

lead to the transformation matrix in the angles between normal vectors of the mirrors on the mirror corner:

$$
A_{\text {lin }}=\left(\begin{array}{ccc}
1 & -\Delta \varphi_{x y} & -\Delta \varphi_{x z} \\
0 & 1 & \Delta \varphi_{y z} \\
0 & 0 & 1
\end{array}\right) .
$$

If $\varphi_{i j}>90^{\circ}$ the angles between the mirror normal angles are smaller than $90^{\circ}$. Figure 3 shows the following case:

- Angle between $x$-mirror normal vector and $y$-mirror normal vector $<90^{\circ}$ resp. angle between $x$-mirror and $y$-mirror $>90^{\circ} . \Delta \varphi_{x y}<0$

- Angle between $x$-mirror normal vector and $z$-mirror normal vector $<90^{\circ}$ resp. angle between $x$-mirror and $z$-mirror $>90^{\circ} . \Delta \varphi_{x z}<0$

- Angle between $y$-mirror normal vector and $z$-mirror normal vector $<90^{\circ}$ resp. angle between $y$-mirror and $z$-mirror $>90^{\circ} . \Delta \varphi_{y z}<0$ 


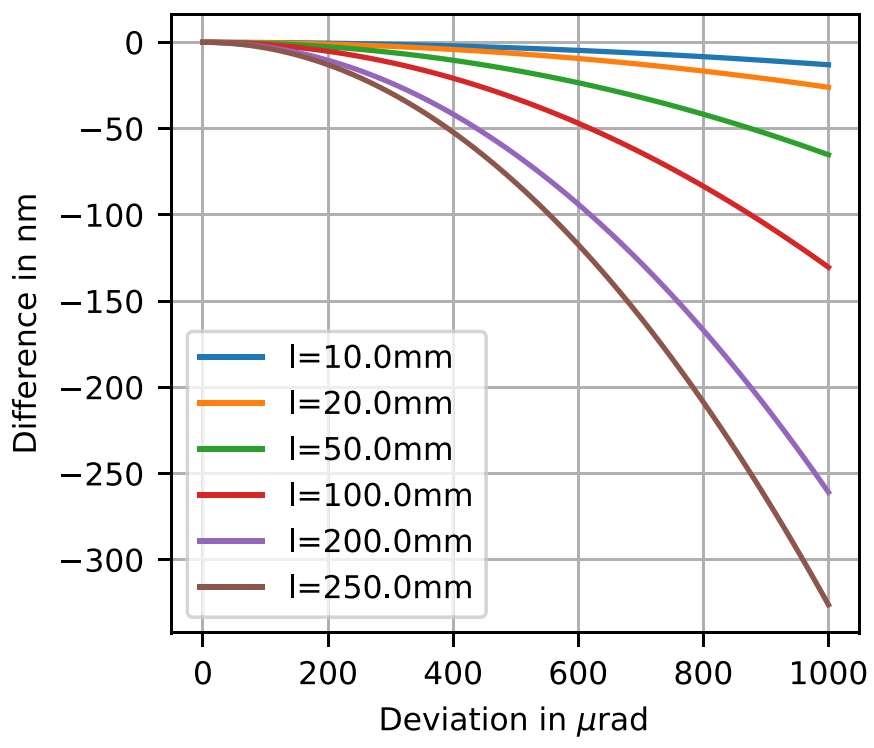

Figure 6. Difference in length measurement $\Delta l_{\text {lin }}$ between the correct coordinate transformation and the linearised transformation, dependent on the angle between the base vectors

$\Delta \varphi=\Delta \varphi_{12}=\Delta \varphi_{13}=\Delta \varphi_{23}$ for different measurement distances.

\subsection{Comparison of coordinate transformations}

The different transformation matrices $A_{\text {cor }}, A_{\text {sim }}$, and $A_{\text {lin }}$ (see equations (17) (23) (24)) are compared with a simulated set of data. Thereby a measurement vector $\vec{r}$, which goes diagonally through the measurement volume in the orthogonal coordinate system of the NPMM, is used:

$$
\vec{r}=\left(x_{O}, y_{O}, z_{O}\right)=(200 \mathrm{~mm}, 200 \mathrm{~mm}, 25 \mathrm{~mm}) .
$$

Its length $l$ can be calculated directly:

$$
|\vec{r}|=l=\sqrt{x_{O}^{2}+y_{O}^{2}+z_{O}^{2}}
$$

This vector is transformed into the skewed coordinate system by the correct transformation matrix in order to calculate the components which would have been measured by the interferometers in covariant coordinates:

$$
\vec{r}=\left(x_{s}, y_{s}, z_{s}\right)=A_{\mathrm{cor}}^{-1} \cdot\left(\begin{array}{c}
x_{O} \\
y_{O} \\
z_{O}
\end{array}\right)=a_{l}^{m} \cdot\left(\begin{array}{c}
x_{O} \\
y_{O} \\
z_{O}
\end{array}\right) .
$$

Afterwards, these coordinates are transformed back with the simplified and the linearised transformation matrix:

$$
\vec{r}_{\text {sim }}=A_{\text {sim }} \cdot \vec{r} \quad \vec{r}_{\text {lin }}=A_{\text {lin }} \cdot \vec{r}
$$

and the difference in length of the vector is calculated:

$$
\Delta l_{\text {sim }}=\left|\vec{r}_{\text {sim }}\right|-|\vec{r}| \quad \Delta l_{\text {lin }}=\left|\vec{r}_{\text {lin }}\right|-|\vec{r}| \text {. }
$$

As a worst-case estimation all angles between the base vectors are varied between $0 \mu \mathrm{rad} \leq \Delta \varphi \leq \mathrm{mm} 1000 \mu \mathrm{rad}$ :

$$
\Delta \varphi=\Delta \varphi_{12}=\Delta \varphi_{13}=\Delta \varphi_{23} .
$$

Even for very large angular deviations $(1000 \mu \mathrm{rad})$ the difference between the simplified and the linearised transformation is below $0.028 \mathrm{~nm}$ for a length of $l=250 \mathrm{~mm}$. Since this difference is much smaller than the achievable uncertainty with the NPMM-200, only the linearised transformation matrix is compared with the correct transformation matrix.

Figure 6 shows the difference between the correct transformation and the linearised transformation matrix dependent on the angle $\Delta \varphi$. The difference between the correct length and the linearised results increases significantly with rising non-orthogonality. For angular deviations below mm50 $\mu \mathrm{rad}$, the difference in length stays below $\mathrm{mm} 1 \mathrm{~nm}$.

\subsection{GUM-based uncertainty}

For further examination, the overall measurement uncertainty is calculated following the rules of the guide to the Expression of Uncertainty in Measurement (GUM) [11]. Thereby the length $l$ of the vector $\vec{r}$ is a function $l\left(x_{\mathrm{i}}\right)$ of different input quantities $x_{\mathrm{i}}$. Those input quantities are, for example, the length measurement itself, which is influenced by nonlinearities of the interferometers and changes in the refractive index of air, or the discussed deviations of the orthogonal coordinate system of the mirror corner. Without any correlations the associated uncertainty $u(l)$ of the length measurement can be calculated directly:

$$
u(l)=\sqrt{\sum_{i=1}^{n}\left(\frac{\partial l}{\partial x_{i}} \cdot u\left(x_{i}\right)\right)^{2}} .
$$

Thereby $n$ is the number of input quantities. The deviations of the orthogonal coordinate system have been measured in different experiments and the non-perpendicularity of the NPMM-200 mirror corner is maximally mm16.77 $\mu \mathrm{rad}$ with an uncertainty of $u(\varphi)=\mathrm{mm} 0.14 \mu \mathrm{rad}(\mathrm{k}=2)$ [9]. Based on these values, the influence of the different coordinate transformation is determined. In table 1 the deviation and uncertainties are given for all three angles. The complete uncertainty model with all input quantities is given in [7, 8]. All input quantities beside the angular deviation of the coordinate system are neglected and the value of the length measurement itself is assumed to have no uncertainty. As shown in section 5.1 the measurement vector $\vec{r}$ is transformed into the skewed coordinate system and is transformed back with uncertain transformation angles. So the length $l$ is $283.945417 \mathrm{~mm}$ with an uncertainty of $u(l)=14.2 \mathrm{~nm}(k=2)$, where $k$ is the coverage factor for the uncertainty calculation [11]. Thereby $\varphi_{12}$ has the highest influence on the uncertainty (see uncertainty budget in table 1).

The length and the uncertainty are calculated, as well, for the simplified and linearised coordinate transformation. For both, the difference in length $\Delta l_{\text {sim }}$ and $\Delta l_{\text {lin }}$ is $-58 \mathrm{pm}$ (see table 2). The difference in the uncertainty is negligiblly small, $\Delta u(l)=0.300 \mathrm{pm}$. All coordinate transformations show a comparable expanded uncertainty of $\approx 14.2 \mathrm{~nm}$ which is 
Table 1. Uncertainty budget for the correct coordinate transformation matrix for a simulated measurement vector in the skewed coordinate system $\left(x_{S}, y_{S}, z_{S}\right)$.

\begin{tabular}{llll}
\hline$x_{\mathrm{i}}$ & Value & Uncertainty & Contribution \\
\hline$\Delta \varphi_{12}$ & $16.77 \mu \mathrm{rad}$ & $0.07 \mu \mathrm{rad}$ & $6.83 \mathrm{~nm}$ \\
$\Delta \varphi_{13}$ & $11.19 \mu \mathrm{rad}$ & $0.07 \mu \mathrm{rad}$ & $1.28 \mathrm{~nm}$ \\
$\Delta \varphi_{23}$ & $-4.75 \mu \mathrm{rad}$ & $0.05 \mu \mathrm{rad}$ & $1.28 \mathrm{~nm}$ \\
$x_{S}$ & $199.998827 \mathrm{~mm}$ & $0 \mathrm{~nm}$ & $0.0 \mathrm{~nm}$ \\
$y_{S}$ & $199.999665 \mathrm{~mm}$ & $0 \mathrm{~nm}$ & $0.0 \mathrm{~nm}$ \\
$z_{S}$ & $25.000000 \mathrm{~mm}$ & $0 \mathrm{~nm}$ & $0.0 \mathrm{~nm}$ \\
& $l=283.945417 \mathrm{~mm} \pm 14.2 \mathrm{~nm}(k=2)$ &
\end{tabular}

Table 2. Calculated length for the different coordinate transformations (correct, simplified, linear) and the associated uncertainties.

\begin{tabular}{lll}
\hline Transformation & Length $(l)$ & $\begin{array}{l}\text { Expanded uncertainty } \\
(k=2)\end{array}$ \\
\hline Correct & $283.945417290 \mathrm{~mm}$ & $14.131676 \mathrm{~nm}$ \\
Simplified & $283.945417232 \mathrm{~mm}$ & $14.131376 \mathrm{~nm}$ \\
Linear & $283.945417232 \mathrm{~mm}$ & $14.131376 \mathrm{~nm}$ \\
\hline
\end{tabular}

much larger than the difference in length between these three coordinate transformations.

\subsection{Conclusion}

As is shown in figure 6, and by comparison of the uncertainty budgets (see tables 1 and 2 ), the difference in length between the simplified transformation and the correct coordinate transformation is smaller than the errors which occur from the measurement uncertainty of the mirror normal vectors. Hence, the linearised coordinate transformation can be used without a significant influence on the measurement result and the corresponding expanded uncertainty, as long as the non-orthogonality stays within the measured range. The expanded uncertainty (see table 2) is given with more digits compared to the advice of the GUM in order to show that there is a difference in uncertainty between the used models.

Since the linearised coordinate transformation can be faster in terms of computation time it opens the door for faster real-time control applications in the NPMM-200. This can increase the measurement performance without influencing the uncertainty budget significantly. For current and future nanofabrication tasks, which are performed on the NPMM200, the real-time control performance is of the highest importance $[12,13]$.

\section{Summary}

A systematic approach was used to determine the correct transformation matrix for the covariant coordinates measured by the interferometers of the NPMM-200. This transformation matrix is further simplified and linearised. A GUM uncertainty budget shows that there is no significant difference in the cor- rected length and the corresponding uncertainty by using the linearised coordinate transformation.

\section{Acknowledgments}

The authors gratefully acknowledge the support by the Deutsche Forschungsgemeinschaft (DFG) in the framework of the Research Training Group 'Tip- and laser-based 3DNanofabrication in extended macroscopic working areas' (GRK 2182) at the Technische Universität Ilmenau, Germany.

\section{ORCID iD}

Florian Fern (D) https://orcid.org/0000-0002-1159-0670

\section{References}

[1] Henselmans R, Cacace L A, Kramer G F Y, Rosielle P C J N and Steinbuch M 2011 The nanomefos non-contact measurement machine for freeform optics Precision Eng. 35 607-24

[2] Panasonic 2015 Ultrahigh Accurate 3D Profilometer Datasheet

[3] Widdershoven I, Donker R L and Spaan H A M 2011 Realization and calibration of the Isara 400 ultra-precision CMM J. Phys. Conf. Series 311012002

[4] Bos E, Moers T and Riel M van 2015 Design and verification of an ultra-precision 3D-coordinate measuring machine with parallel drives Meas. Sci. Technol. 26085904

[5] Jäger G, Manske E, Hausotte T, Müller A and Balzer F 2016 Nanopositioning and nanomeasuring machine NPMM-200 - a new powerful tool for large-range microand nanotechnology Surface Topography Metrol. Properties 4034004

[6] Bryan J B 1979 The Abbé principle revisited: An updated interpretation Precision Eng. 1 129-32

[7] Füß1 R, Grünwald R, Rahneberg I and Kreutzer P 2009 Uncertainty consideration of the coordinate system in nanopositioning- and nanomeasuring

machinesunsicherheitsüberlegungen zum

koordinatensystem in nano-positionier- und messmaschinen tm - Technisches Messen 76 235-8

[8] Hausotte T, Percle B, Gerhardt U, Dontsov D, Manske E and Jäer G 2012 Interference signal demodulation for nanopositioning and nanomeasuring machines Meas. Sci. Technol. 23074004

[9] Haifeng X Hochpräzise Bestimmung der Form- und Orthogonalitätsabweichungen einer Spiegelecke und Untersuchung des Verhaltens unter veränderlichen Umweltbedingungen Dissertation Universitäsverlag Ilmenau, Ilmenau

[10] Iben H K and Manteuffel K 1999 Tensorrechnung Tensorrechnung 2nd edn (Wiesbaden: Vieweg+Teubner Verlag)

[11] BIPM 1995 Genève: BIPM Guide to the Expression of Uncertainty in Measurement

[12] Kuehnel M, Fröhlich T, Füßl R, Hoffmann M, Manske E, Rangelow Ivo W, Reger J, Schäffel C, Sinzinger S and Zöllner J-P 2018 Towards alternative 3D nanofabrication in macroscopic working volumes Meas. Sci. Technol. 29 114002

[13] Weidenfeller L, Schienbein R, Reinhardt C, Kirchner J and Manske E 2018 Development of laser positioning system of high accuracy in the nanometer range Proc. SPIE 10544 $105440 \mathrm{E}$ 